TRANSACTIONS OF THE

AMERICAN MATHEMATICAL SOCIETY

Volume 350, Number 8, August 1998, Pages 3065-3081

S 0002-9947(98)01780-2

\title{
LIOUVILLIAN INTEGRATION AND BERNOULLI FOLIATIONS
}

\author{
D. CERVEAU AND P. SAD
}

\begin{abstract}
Analytic foliations in the 2-dimensional complex projective space with algebraic invariant curves are studied when the holonomy groups of these curves are solvable. It is shown that such a condition leads to the existence of a Liouville type first integral, and, under "generic" extra conditions, it is proven that these foliations can be defined by Bernoulli equations.
\end{abstract}

\section{INTRODUCTION}

This paper is devoted to the study of ordinary differential equations (O.D.E.'s) $y^{\prime}=R(x, y)$ where $R$ is a rational function in the complex variables $(x, y)$. There are many contributions in this area-the most famous contributors are Cauchy, Darboux, Briot-Bouquet, Boutroux, Hermite, Jacobi, Liouville, Painlevé, Poincaré, Malmquist and many others - and recently some results appeared with new points of view: local and global theory of dynamical systems, and differential fields theory. In this paper we want to give a bridge between these two approaches and to give also a tentative answer to the following question:

Why in classical books are only such O.D.E.'s as the linear, Bernoulli and Riccati (particular) equations considered?

To the (O.D.E.) $E: y^{\prime}=R(x, y)$ can be associated a polynomial Pfaffian form $\omega_{E}$ in the affine space $\mathbf{C}^{2}$ :

$\omega_{E}:=A d x+B d y, \quad$ g.c.d. $(A, B)=1$, with $R=-A / B$ and $A, B \in \mathbf{C}[x, y]$.

Note that $\omega_{E}$ is well defined up to a multiplicative constant.

The singularities of $\omega_{E}$ are the points of the set $\operatorname{Sing} \omega_{E}$ (or Sing $E$ ) defined by $A=B=0$. The set $\mathbf{C}^{2}-\operatorname{Sing} E$ is naturally foliated by $\omega_{E}$ : the leaves are the solutions of $\omega_{E}=0$; at a generic point $\left(x_{0}, y_{0}\right)$ the local leaf $\mathcal{L}\left(x_{0}, y_{0}\right)$ passing by $\left(x_{0}, y_{0}\right)$ is the graph of the local solution $y(x)$ of $E$ with the initial condition $y\left(x_{0}\right)=y_{0}$. To describe this foliation it is often convenient to construct a reasonable compactification $M_{E}$ of $\mathbf{C}^{2}-\operatorname{Sing} E$ with an holomorphic extension of our foliation with singularities; in general this compactification appears as a ruled surface. In the classical books concerning elementary theory of O.D.E.'s, the strategy is quasi universal (for example see traditional books as Ince, Valiron or Petrovski). It is important to have in mind this folklore which gives some examples of compactifications $M_{E}$.

1. Equations with separated variables: $E: y^{\prime}=-P_{1}(x) / P_{2}(y), P_{i} \in \mathbf{C}(t)$. The rational form $\alpha=P_{1}(x) d x+P_{2}(y) d y$ is closed and can be integrated by using the standard decomposition of rational functions; we obtain a first integral $F$ of our

Received by the editors December 7, 1994 and, in revised form, November 28, 1995.

1991 Mathematics Subject Classification. Primary 32L30; Secondary 58F18. 
equation in an elementary extension of $\mathbf{C}(x, y)$ (see $\S 5$ below), $F=\sum \lambda_{i} \log \nu_{i}+v$ with $u_{i}$ and $v$ in $\mathbf{C}(x, y), \lambda_{i} \in \mathbf{C}$. The Pfaffian form $\omega_{E}$ is equal to

$$
\omega_{E}=q_{1}(x) \cdot q_{2}(y) \cdot \alpha, \quad q_{i} \in \mathbf{C}[t] \text { are the denominators of the } P_{i} \text { 's, }
$$

and $\omega_{E}$ has the integrating factor $f=q_{1} \cdot q_{2}$ :

$$
d\left(\frac{\omega_{E}}{f}\right)=0 \text { or equivalently } d \omega_{E}=\frac{d f}{f} \wedge \omega_{E} .
$$

Note that the closed rational 1-form $\eta$ has simple poles with integral residues. For this type of equation it is natural to consider the compactification $\mathbf{P C}(1) \times$ $\mathbf{P C}(1)=M_{E}$. Note also that the foliation $\mathcal{F}_{E}$ induced by $E$ on $M_{E}$ is transversal to the natural fibrations by horizontal (resp. vertical) lines except along some special horizontal (resp. vertical) lines which are the closures of particular leaves of $\mathcal{F}_{E}$.

2. Homogeneous equations: $E: y^{\prime}=f\left(\frac{y}{x}\right), f \in \mathbf{C}(t)$. In this case Valiron (and others) says: put $t=y / x$, and in the new coordinates "c'est une équation à variable séparée qui peut s'écrire:

$$
\frac{d x}{x}=\frac{d t}{f(t)-t} "
$$

here the adapted compactification $M_{E}$ is obtained by blowing up a point (the origin in the affine chart $\mathbf{C}^{2}$ ) in the projective space $\mathbf{C P}(2)$. This space is naturally a fibration (with fibers isomorphic to $\mathbf{C P}(1)$ ) and the foliation $\mathcal{F}_{E}$ is transversal to the fibers except for a finite number of them which are, as above, the closures of particular leaves of $\mathcal{F}_{E}$. As previously the Pfaffian form $\omega_{E}$ has a rational integrating factor and an elementary first integral.

3. Linear and Bernouilli equations. Linear equations are of type $E: y^{\prime}+$ $A(x) y+B(x)=0$ for $A, B \in \mathbf{C}(t)$; the compactification of $\mathbf{C}^{2}$ by $\mathbf{C P}(1) \times \mathbf{C P}(1)$ is well adapted; note that the change of variables $y \mapsto \frac{1}{y}$ in the vertical fibers gives us the new equation:

$$
-y^{\prime}+y A(x)+y^{2} B(x)=0
$$

which is a special type of Riccati equation. The foliation induced by $E$ in $\mathbf{C P}(1) \times$ $\mathbf{C P}(1)$ is transversal to the vertical fibration except along a finite set of lines (the poles of $A$ and $B$ ). The differential form in the affine space $\mathbf{C}^{2}$ is of the type

$$
\omega_{E}=c(x) d y+(a(x) y+b(x)) d x, \quad a, b, c \in \mathbf{C}[x],
$$

and in general $\omega_{E}$ has no rational integrating factor, as in the previous cases. But if we try to compute such an $f$ we see that:

$$
d \omega_{E}=\left(\frac{d c}{d x}-a\right) d x \wedge d y=\left(\frac{d c}{d x}-a\right) \cdot c^{-1} d x \wedge \omega_{E}
$$

and the closed rational form $\eta=\left(\frac{d c}{d x}-a\right) c^{-1} . d x$ satisfies:

$$
d \omega_{E}=\eta \wedge \omega_{E}, \quad d \eta=0
$$

If we write $\eta=\frac{d F}{F}$, with $F=e^{r} r_{1}^{\lambda_{1}} \cdots r_{p}^{\lambda_{p}}, r, r_{i} \in \mathbf{C}(x), \lambda_{i} \in \mathbf{C}$, we see that $F$ is an elementary (multivalued) integrating factor of $\omega_{E}$; in general the $\lambda_{i}$ 's are not integers. 
Bernouilli equations $E: y^{\prime}+A(x) \cdot y+B(x) \cdot y^{n}=0, n \in \mathbf{N}$ and $A, B \in \mathbf{C}(x)$. If $n \in\{0,1,2\}$ we are in the previous cases; if it is not the case, $E$ appears as a ramification (put $Y=y^{1-n}$ ) of a linear equation and as a consequence there exists a closed rational form $\eta$ satisfying $(*)$ and $E$ has an elementary first integral.

4. General Riccati equation. Classical books end the elementary study of O.D.E.'s by the Riccati equation $E: y^{\prime}+A(x) y^{2}+B(x) y+C(x)=0, A, B, C \in \mathbf{C}[x]$.

Valiron says: "Liouville a montré qu'elle ne s'intègre pas en général..." The natural compactification here is $\mathbf{C P}(1) \times \mathbf{C P}(1)$ and the above affirmation can be justified by using differential Galois theory applied to the linear differential equation of second order associated to $E$; this equation is constructed by introducing a new variable $z$ defined by $y=-\frac{z^{\prime}}{z A}$. We shall make all this precise by using Singer's results.

5. Elementary and Liouvillian extensions. We consider $K_{0}=\mathbf{C}(x, y)$ as a differential field with the set of derivations $\Delta=\left(\frac{\partial}{\partial x}, \frac{\partial}{\partial y}\right)$; an elementary extension $K$ of $K_{0}$ is a differential field obtained as follows; there exists a tower of differential fields $\left(K_{i}, \Delta_{i}\right), i=1, \ldots, n$, such that:

1. $K_{0} \subset K_{1} \subset \cdots \subset K_{n}=K$.

2. $\left.\Delta_{i}\right|_{K_{i-1}}=\Delta_{i-1}$ so we note $\Delta_{i}=\left(\frac{\partial}{\partial x}, \frac{\partial}{\partial y}\right)$.

3. The field of constants $C\left(K_{i}\right)=\left\{f \in K_{i}, \delta f=0 \forall \delta \in \Delta_{i}\right\}=C\left(K_{0}\right)=\mathbf{C}$.

4. $K_{i}=K_{i-1}\left(t_{i}\right)$ where $t_{i}$ is one of the following 3 types:

4.a. $t_{i}$ is algebraic over $K_{i-1}$,

4.b. there exists $a \in K_{i-1}$ s.t. $\delta\left(t_{i}\right)=\frac{\delta(a)}{a}, \forall \delta \in \Delta$,

4.c. there exists $a \in K_{i-1}$ s.t. $\frac{\delta\left(t_{i}\right)}{t_{i}}=\delta a, \forall \delta \in \Delta$.

Remark. If $f$ is an element in $K$, by construction $f$ can be seen as an analytic function on a certain open set $U_{f}$ on $\mathbf{C}^{2}$.

Condition 4.b says that we add the logarithm of a certain element in $K_{i-1}$ and 4.c that we add the exponential of an element.

Now we say that the differential field $K$ is a Liouvillian extension of $K_{0}=\mathbf{C}(x, y)$ if $K$ verifies conditions $1,2,3,4$.a, or 4 .c or

4. $\mathrm{b}^{\prime} \forall \delta \in \Delta$ there exists $a_{\delta} \in K_{i-1}$ such that $\delta\left(t_{i}\right)=a_{\delta}$.

The condition $4 . \mathrm{b}^{\prime}$ permits us to add the primitive of a closed differential form $a d x+b d y, \frac{\partial b}{\partial x}=\frac{\partial a}{\partial y}$, with coefficients $a$ and $b$ in $K_{i-1}$.

Note that an elementary extension is Liouvillian, but the converse is not true.

In the same way we can define elementary and Liouvillian extensions of $\left(\mathbf{C}(x), \frac{\partial}{\partial x}\right)$. We introduce now the two following definitions:

Definition 1. We say that $E: y^{\prime}=R(x, y), R \in \mathbf{C}(x, y)$, has a Liouvillian solution if there exist a Liouvillian extension $\left(L, \frac{\partial}{\partial x}\right)$ of $\left(\mathbf{C}(x), \frac{\partial}{\partial x}\right)$ and an element $l \in L$ satisfying $\frac{\partial l}{\partial x}=R(x, l)$.

Definition 2. $E: y^{\prime}=R(x, y)=-A(x, y) / B(x, y)$ has a Liouvillian (resp. elementary) first integral if there exist a Liouvillian (resp. elementary) extension $\left(K,\left(\frac{\partial}{\partial x}, \frac{\partial}{\partial y}\right)\right)$ of $\mathbf{C}(x, y)$ and an element $f \in K$ such that

$$
\omega_{E} \wedge d f=0, \text { i.e. if } \omega_{E}=A d x+B d y, \quad A \frac{\partial f}{\partial y}-B \frac{\partial f}{\partial x}=0 .
$$

We now present some results of Singer (see [13]): 
Theorem 1. Suppose $E: y^{\prime}=R(x, y)$ has a Liouvillian first integral. Then there exists a closed rational 1-form $\eta$ such that $d \omega_{E}=\eta \wedge \omega_{E}$. Conversely if such an $\eta$ exists then $E$ has a Liouvillian first integral.

Recall that a rational closed 1-form $\eta$ is of the following type [4]:

$$
\eta=\sum_{j=1}^{p} \lambda_{j} \frac{d P_{j}}{P_{j}}+d\left(\frac{H}{P_{1}^{n_{1}} \cdots P_{p}^{n_{p}}}\right), \quad n_{i} \in \mathbf{N}, \lambda_{i} \in \mathbf{C}^{*}, H, P_{j} \in \mathbf{C}[x, y] .
$$

The algebraic curves $P_{j}=0$ are the poles of $\eta$ and the $\lambda_{j}$ 's are the residues of $\eta$ along $P_{j}=0$ :

$$
\lambda_{j}=\frac{1}{2 \pi i} \int_{\gamma_{j}} \eta
$$

where $\gamma_{j}$ are small circles around $P_{j}=0$. As a consequence the elementary function $F=e^{H /\left(P_{1}^{n_{1}} \cdots P_{p}^{n_{p}}\right)} P_{1}^{\lambda_{1}} \cdots P_{p}^{\lambda_{p}}$ is an integrating factor of $\omega_{E}$.

In fact Singer also proves

Theorem 2. Suppose that $E: y^{\prime}=R(x, y)$ has a Liouvillian solution $l$. Then either $l$ is algebraic in $\mathbf{C}(x)$ (and there exists an algebraic curve $\Gamma$-which coincides in an open set with the graph of $l$-such that $\Gamma$ is the closure of a leaf of $\left.\mathcal{F}_{E}\right)$ or $E$ has a Liouvillian first integral.

For elementary first integrals we have (see [10]):

Theorem 3 (Prelle-Singer). If $E$ has an elementary first integral, it has a super elementary first integral, that is, of type $\sum \mu_{i} \log P_{i}+Q, \mu_{i} \in \mathbf{C}, P_{i}$ and $Q$ algebraic over $\mathbf{C}[x, y]$.

Let us explain the objective of this paper. Firstly, we start with a dynamical condition on the foliation in $\mathbf{C P}(2)$ associated to the O.D.E.; namely, there exists a leaf whose closure is an algebraic curve and whose holonomy group is a nonabelian solvable one. This means that the group is a ramification of a subgroup of Moebius transformations. We raise then the problem of classification of these foliations, which is a natural question since we already know its answer when the holonomy group is abelian. Now the dynamical condition translates to the same condition of Singer's theorem, namely $d \omega_{E}=\eta \wedge \omega_{E}$; a subsequent analysis leads to the strong conclusion (in the generic case) that we are in the presence of a Bernoulli equation. We think this is the reason why such an equation predominates in classical books.

Before ending the Introduction, let us say a word about Liouville's statement of "generic nonintegrability" of Riccati equations $E: y^{\prime}+A(x) y^{2}+B(x) y+C(x)=0$, $A, B, C \in \mathbf{C}(x)$. Write $\omega_{E}=p(x) d y+\left(a(x) y^{2}+b(x) y+c(x)\right) d x, a, b, c, p \in \mathbf{C}[x]$ and suppose now that there exists a closed rational form $\eta$ such that:

$$
d \omega_{E}=\eta \wedge \omega_{E} .
$$

By Theorem 2, this hypothesis is satisfied if $E$ has a Liouvillian solution. Note that each irreducible component of the divisor of poles of $\eta$ is a separatrix for the foliation $\mathcal{F}_{E}$ associated to $\omega_{E}$. The classical differential Galois theory applied to some adapted second order linear O.D.E. (see above) shows that in general $E$ does not have algebraic solutions (see Kaplansky [7]). This fact can be proved (see [7]) also by direct computation (overdeterminated systems) or by geometric arguments involving Tits' alternative [14] and the realization of finitely generated subgroup of 
the Moebius group as holonomy groups of Riccati equations [8]. This implies that generically the poles of $\eta$ are vertical lines $x=x_{i}$ so that

$$
\eta=\sum_{i=1}^{p} \lambda_{i} \frac{d x}{x-x_{i}}+d \frac{H}{\prod\left(x-x_{i}\right)^{n_{i}}}, \quad H \in \mathbf{C}[x, y], n_{i} \in \mathbf{N}, \lambda_{i} \in \mathbf{C}^{*} .
$$

Direct computation of $d \omega_{E}$ and condition $(*)$ implies:

$$
\begin{aligned}
d \omega_{E}= & \left(\frac{\partial p}{\partial x}-2 y a-b\right) \frac{d x}{p} \wedge \omega_{E}=\left(\sum \frac{\lambda_{i}}{x-x_{i}}+\frac{\partial}{\partial x}\left(\frac{H}{\prod\left(x-x_{i}\right)^{n_{i}}}\right)\right) p(x) \\
& -\frac{\partial H}{\partial y}\left(\prod\left(x-x_{i}\right)^{n_{i}} \cdot\left(a(x) y^{2}+b(x) y+c(x)\right)\right)
\end{aligned}
$$

and since $H$ is polynomial $\frac{\partial H}{\partial y}=0$ if $a \neq 0$. But this implies $a=0$; contradiction. So we obtain:

If the Riccati equation $y^{\prime}=\frac{a(x) y^{2}+b(x) y+c(x)}{p(x)}, a, b, c, p \in \mathbf{C}[x]$, has a Liouvillian solution then it has a solution which is algebraic over $\mathbf{C}[x]$. And generically Riccati equations don't have algebraic solutions.

This fact is well known, but it is interesting to recall it because as we said the condition $(*)$ has a dynamic interpretation.

\section{Solvable holONOMY GROUPS AND FOLiations}

Let us consider a holomorphic foliation $\mathcal{F}$ in $\mathbf{C} P(2)$ of degree $\nu \in \mathbf{N}, \nu \geq 2$, such that:

(i) there exists a smooth algebraic separatrix $S \subset \mathbf{C P}(2)$, along which $\mathcal{F}$ has simple singularities $p_{0}, \ldots, p_{m}$, one of them at least hyperbolic (by a simple singularity it is meant a local expression as $x d y-\lambda y d x+$ h.o.t. $=0$; when $\lambda \notin \mathbf{R}_{+} \cup\{0\}$ we say that the singularity is hyperbolic).

(ii) The holonomy group $\mathcal{H}$ of $S \backslash\left\{p_{0}, \ldots, p_{m}\right\}$ is a solvable group.

The group $\mathcal{H}$ can be described according to the following two possibilities.

- $\mathcal{H}$ is an abelian group. Since it contains a hyperbolic attractor by hypothesis, $\mathcal{H}$ can be made linear in some appropriate coordinate and therefore it is possible to construct a Darboux integral for $\mathcal{F}$ (see [1]).

- $\mathcal{H}$ is a nonabelian group. We define for $p \in \mathbf{N}$ :

$$
\begin{aligned}
& \mathbf{H}_{1}=\left\{z \mapsto \frac{a z}{1+b z}, a \in \mathbf{C}^{*}, b \in \mathbf{C}\right\}, \\
& \mathbf{H}_{p}=\left\{z \mapsto \frac{a z}{(1+b z)^{1 / p}}, a \in \mathbf{C}^{*}, b \in \mathbf{C},(1)^{1 / p}=1\right\} .
\end{aligned}
$$

Then $\mathcal{H}$ is holomorphically conjugated to a subgroup of $\mathbf{H}_{p}$ for some $p \in \mathbf{N}$ (see [5]); we say that $p \in \mathbf{N}$ is the ramification order of $\mathcal{H}$ (by the way, we remark that any subgroup of $\mathbf{H}_{p}$ is solvable).

Let us give two examples.

(i) Riccati foliations: the algebraic separatrix is a projective line $L$ which we regard as the line at infinity of $\mathbf{C} P(2)$. In affine coordinates $(z, w) \in \mathbf{C}^{2}$ the foliation $\mathcal{F}_{1}$ of degree $\nu \in \mathbf{N}$ is defined by $\omega=0$, where

$$
\omega=f_{\nu-2}(z, w)(z d w-w d z)+p(z, w) \sum_{k=0}^{\nu} \lambda_{k} \frac{d\left(w-a_{k} z\right)}{w-a_{k} z} ;
$$


here $f_{\nu-2}$ is a homogeneous polynomial of degree $\nu-2(\nu \geq 2)$

$$
\begin{gathered}
p(z, w)=\prod_{k=0}^{\nu}\left(w-a_{k} z\right), \\
\sum_{k=0}^{\nu} \lambda_{k}=1, \lambda_{k} \neq 0, a_{j} \neq a_{k} \text { if } j \neq k .
\end{gathered}
$$

The origin $(0,0) \in \mathbf{C}^{2}$ is a dicritical singularity, contained in the separatrices $L_{k}$ : $w-a_{k} z=0, k=0, \ldots, \nu$. There is also another separatrix $L$ as the line at infinity, whose intersections with $L_{0}, \ldots, L_{\nu}$, are the singularities of $\left.\mathcal{F}_{1}\right|_{L}$, and the holonomy group $\mathcal{H}$ of $L \backslash \operatorname{Sing} \mathcal{F}_{1}$ is a subgroup of $\mathbf{H}_{1}$. In order to see this, we change the affine coordinates to have $L$ as the horizontal straight line $\overline{\{y=0\}}$ and the new line at infinity not invariant for $\mathcal{F}_{1}$. Then $\mathcal{F}_{1}$ is given by the equation

$$
p(x) d y-\left(P(x) y^{2}+Q(x) y\right) d x=0
$$

where $p(x)=p(1, x), P(x)=f_{\nu-2}(1, x), Q(x)=p(x) \sum_{k=0}^{\nu} \frac{\lambda_{k}}{x-a_{k}}$. Besides $L$, the other separatrices are the vertical lines $L_{k}=\overline{\left\{x-a_{k}=0\right\}}, k=0, \ldots, \nu$, and the points $\left(a_{k}, 0\right), k=0, \ldots, \nu$, are the singularities of $\left.\mathcal{F}_{1}\right|_{L}$. Since $(*)$ defines a foliation of $\overline{\mathbf{C}} \times \overline{\mathbf{C}}$ transversal to the vertical lines (except for the separatrices), we see that $\mathcal{H}$ is a subgroup of $\mathbf{H}_{1}$.

(ii) Bernoulli foliations: Let us fix affine coordinates $(x, y) \in \mathbf{C}^{2}$ and consider the Riccati foliation $\mathcal{F}_{1}$

$$
p(x) d y-\left(P(x) y^{2}+Q(x) y\right) d x=0,
$$

where $p, P$ and $Q$ are polynomials as before. If $\phi$ denotes the rational transformation of $\mathbf{C} P(2)$ :

$$
(x, y) \mapsto \phi(x, y)=\left(x, y^{p}\right), \quad p \in \mathbf{N},
$$

the foliation $\mathcal{F}_{p}=\phi^{*} \mathcal{F}_{1}$ is given by

$$
p \cdot p(x) d y-\left(P(x) y^{p+1}+Q(x) y\right) d x=0 .
$$

The holonomy group of $L \backslash \operatorname{Sing} \mathcal{F}_{p}$ is a $p$-ramification of a subgroup of $\mathbf{H}_{1}$, and therefore it is a subgroup of $\mathbf{H}_{p}$.

It is an open problem to classify the foliations in $\mathbf{C} P(2)$ which have an invariant algebraic curve whose holonomy group is solvable. We study here the "generic" case, although the techniques employed seem to be useful in general. There are also interesting results in [11].

\section{Transverse AfFine Structures}

It is well known (see [12] for example) that a foliation defined by a differential 1form $\Omega=0$ satisfying $d \Omega=\mathcal{N} \wedge \Omega$ for some closed 1-form $\mathcal{N}$ is naturally associated to a transverse affine structure. This fact is used here with some adaptations to be explained now; they are necessary since we deal with meromorphic 1-forms.

Let $\mathcal{F}$ be a holomorphic foliation in $\mathbf{C} P(2), \pi: \mathbf{C}^{3} \backslash\{(0,0,0)\} \rightarrow \mathbf{C} P(2)$ be the canonical projection. It can be proved that the foliation $\pi^{*} \mathcal{F}$ is defined by an equation $\Omega=0$ where $\Omega$ is a homogeneous polynomial 1-form of some degree $\nu+1 \epsilon$ $\mathbf{N}$ such that $i_{R}(\Omega)=0$ ( $R$ is the radial vector field in $\left.\mathbf{C}^{3}\right)$. In affine coordinates $(x, y) \in \mathbf{C}^{2}$, the foliation $\mathcal{F}$ is defined by a polynomial 1-form (of degree $\left.\nu+1\right) \omega=0$; the relation $\pi_{1}^{*} \omega=X^{-(\nu+2)} \Omega$ holds, where $\pi_{1}(X, Y, Z)=\left(Y X^{-1}, Z X^{-1}\right)=(x, y)$. 
A meromorphic 1-form $\xi=\sum_{j=1}^{l} a_{j} \frac{d P_{j}}{P_{j}}$, where $P_{1}, \ldots, P_{l}$ are irreducible homogeneous polynomials such that $\xi \wedge \Omega=0$ is said to be a Darboux integral of $\Omega$; $\xi$ is projective since $i_{R}(\Omega)=0$ and $\xi \wedge \Omega=0$ imply $i_{R}(\xi)=0$. We remark that $i_{R}(\xi)=0$ is equivalent to $\sum_{j=1}^{l} a_{j} \operatorname{deg}\left(P_{j}\right)=0$.

Let $\mathcal{A}$ be the set of meromorphic 1 -forms in $\mathbf{C}^{3}$ of the type $\sum_{j=1}^{l} \lambda_{j} \frac{d P_{j}}{P_{j}}$, where $P_{j}$ are irreducible homogeneous polynomials in $\mathbf{C}^{3}$. When $\Omega$ has no Darboux integral, there exists at most one $\mathcal{N} \in \mathcal{A}$ such that $d \Omega=\mathcal{N} \wedge \Omega$. In fact, if $\mathcal{N}_{1}, \mathcal{N}_{2} \in \mathcal{A}$ and $d \Omega=\mathcal{N}_{j} \wedge \Omega$ for $j=1,2$, then $\left(\mathcal{N}_{1}-\mathcal{N}_{2}\right) \wedge \Omega=0$ so $\mathcal{N}_{1}=\mathcal{N}_{2}$.

Now we recall the situation we had in the previous section; the foliation $\mathcal{F}$ in $\mathbf{C} P(2)$ has a smooth algebraic separatrix $S$ with simple singularities whose holonomy group is conjugated to a non abelian subgroup of $\mathbf{H}_{p}$. The following basic result is due to A. Lins Neto (see Appendix).

Theorem 1. There exists an unique $\mathcal{N} \in \mathcal{A}$ such that $d \Omega=\mathcal{N} \wedge \Omega$. More precisely,

$$
\mathcal{N}=(p+1) \frac{d P}{P}+\sum_{j}\left[\operatorname{Res}_{C_{j}}(\mathcal{N})\right] \frac{d P_{j}}{P_{j}},
$$

where $\pi^{-1}(S)=P^{-1}(0), C_{j}=P_{j}^{-1}(0)$ are the separatrices of the foliation given by $\Omega=0 ; P$ and $P_{j}$ are homogeneous polynomials and

$$
(p+1) \operatorname{deg} P+\sum_{j}\left[\operatorname{Res}_{C_{j}}(\eta)\right] \operatorname{deg} P_{j}=\nu+2 .
$$

In fact, this theorem is proven directly in $\mathbf{C} P(2)$, yielding the following conclusion (affine coordinates $(x, y) \in \mathbf{C}^{2}$ are fixed as above, $\bar{P}(x, y)=P(1, x, y), \bar{P}_{j}(x, y)$ $\left.=P_{j}(1, x, y), \pi^{-1}\left(\bar{C}_{j}\right)=C_{j}\right)$ :

1) there exists a closed meromorphic 1-form $\eta$ in $\mathbf{C} P(2)$ such that $d \omega=\eta \wedge \omega, \eta$ can be written as $\eta=(p+1) \frac{d \bar{P}}{P}+\sum_{j} \operatorname{Res}_{\bar{C}_{j}}(\eta) \frac{d \bar{P}}{\bar{P}_{j}}$, so that $\pi_{1}^{*} \eta=\mathcal{N}-(\nu+2) \frac{d X}{X}$

2) $\operatorname{Res}_{L}(\eta)=-(\nu+2), \operatorname{Res}_{\bar{C}_{j}}(\eta)=\operatorname{Res}_{C_{j}}(\mathcal{N})=1-p \operatorname{ind}_{q}(\mathcal{F}, S)$, where $L$ is the line at infinity (supposed to be not invariant for the foliation), $q$ is any of the singularities in $\bar{C}_{j} \cap S$ and $\operatorname{ind}_{q}(\mathcal{F}, S)$ is the index defined in [2].

A statement like this in $\mathbf{C} P(2)$ is more useful, although $\eta$ depends on the affine coordinate system. Once we have the 1 -form $\eta$ we proceed as follows to associate to $\mathcal{F}$ a transverse affine structure:

(a) in $\mathbf{C} P(2) \backslash L \approx \mathbf{C}^{2}$, outside singularities and separatrices of $\mathcal{F}$ : we cover this region $R$ by open sets $U \subset \mathbf{C}^{2}$ where $\left.\mathcal{F}\right|_{U}$ is equivalent to the trivial foliation $\bigcup_{t \in \Gamma_{U}} \mathbf{D} \times\{t\}$, where $\Gamma_{U}$ is a transverse section to $\mathcal{F}$. Then $\eta=d g_{U}$ for some $g \in \mathcal{O}_{U}$. Let $f_{U}:=e^{g_{U}}$, so that $\eta=f_{U}^{-1} d f_{U}$. It follows that

$$
\begin{aligned}
d\left(f_{U}^{-1} \omega\right) & =-f_{U}^{-2} d f_{U} \wedge \omega+f_{U}^{-1} d \omega=-f_{U}^{-2} d f_{U} \wedge \omega+f_{U}^{-1} d \omega \\
& =-f_{U}^{-1}\left(f_{U}^{-1} d f_{U} \wedge \omega\right)+f_{U}^{-1} d \omega=-f_{U}^{-1} \eta \wedge \omega+f_{U}^{-1} d \omega \\
& =-f_{U}^{-1} d \omega+f_{U}^{-1} d \omega=0
\end{aligned}
$$

and so $f_{U}^{-1} \omega=d t_{U}$ for some $t_{U} \in \mathcal{O}_{U}$.

On the other hand, if $U_{1} \cap U_{2} \neq 0$ we have $f_{U_{1}}^{-1} d f_{U_{1}}=f_{U_{2}}^{-1} d f_{U_{2}}$; therefore $f_{U_{2}} f_{U_{2}}^{-1}=$ $c_{U_{1} U_{2}} \in \mathbf{C}^{*}$ (we may assume all the intersections to be connected). Since $\omega=$ $f_{U_{1}}^{-1} d t_{U_{1}}=f_{U_{2}}^{-1} d t_{U_{2}}$ we get $c_{U_{1} U_{2}} d t_{U_{2}}=d t_{U_{1}}$ and $t_{U_{1}}=c_{U_{1} U_{2}} t_{U_{2}}+d_{U_{1} U_{2}}$ in $U_{1} \cap U_{2}$, 
where $d_{U_{1} U_{2}} \in \mathbf{C}$. The collection $\left\{\left(t_{U}, \Gamma_{U}\right)\right\}_{U}$ is the transverse affine structure for $\left.\mathcal{F}\right|_{R}$

(b) in $L$, outside singularities and separatrices of $\mathcal{F}$ : let us use the affine coordinates $(u, v) \in \mathbf{C}^{2}$ where $u=x^{-1}, v=x^{-1} y$; then $\mathcal{F}$ is defined by the polynomial equation $\tilde{\omega}=0, \tilde{\omega}=u^{-(\nu+2)} \omega$. From the theorem stated above we may write $d \tilde{\omega}=\tilde{\eta} \wedge \tilde{\omega}, \tilde{\eta}$ being an appropriate meromorphic 1-form. The relation $d \omega=\left(\tilde{\eta}-(\nu+2) u^{-1} d u\right) \wedge \omega$ follows easily, and we repeat the same argument as in (a). We put $\tilde{\eta}=\tilde{h}^{-1} d \tilde{h}$ in $U\left(\tilde{h} \in \mathcal{O}_{U}^{*}\right)$ and $f_{U}:=u^{-(\nu+2)} \tilde{h}$, so that $f_{U}^{-1} \omega=\tilde{h}^{-1} \tilde{\omega}$ is holomorphic and $d\left(f_{U}^{-1} \omega\right)=0, f_{U}^{-1} d f_{U}=\eta$; $t_{U}$ is defined to satisfy $f_{U}^{-1} \omega=d t_{U}$

(c) in $S$, outside the singularities of $\mathcal{F}$ : let $s \in S$ be a regular point of $\mathcal{F}, \Gamma_{s}$ a transverse section. We may choose a coordinate $\tilde{y} \in \Gamma_{s}$ such that $\eta=(p+1) \frac{d \tilde{y}}{\tilde{y}}=\frac{d\left(\tilde{y}^{p+1}\right)}{\tilde{y}^{p+1}}$ in a neighborhood $U$ of $s \in S$ where $\mathcal{F}$ is trivial; we put $f_{U}:=\tilde{y}^{p+1}$, so $\tilde{y}^{p(p+1)} \omega$ is a closed meromorphic 1 -form. Let $\Gamma_{s}^{(1)}, \Gamma_{s}^{(2)}$ be the complements in $\gamma_{s}$ of two opposite half lines $r e^{i \theta_{0}}, r e^{i\left(\theta_{0}+\pi\right)}$, $0 \leq r \leq r_{0}$, and $U^{(1)}, U^{(2)}$ be obtained by saturating $\Gamma_{s}^{(1)}, \Gamma_{s}^{(2)}$ by $\left.\mathcal{F}\right|_{U}$. We introduce $t_{U^{(1)}}, t_{U^{(2)}}$ in order to have $\tilde{y}^{-(p+1)} \omega=d t_{U^{(1)}}$ in $U^{(1)}$ and $\tilde{y}^{-(p+1)} \omega=d t_{U^{(2)}}$ in $U^{(2)}$, and add to our collection of affine coordinates the elements $\left\{t_{U^{(1)}}, \Gamma_{s}^{(1)}\right\}_{s \in S},\left\{t_{U^{(2)}}, \Gamma_{s}^{(2)}\right\}_{s \in S}$. These coordinates have a special property that will be important for us. Since $\omega=g(\tilde{y}) d \tilde{y}$ in $U$, for some $g \in \mathcal{O}_{U}$, from $\tilde{y}^{-(p+1)} \omega=d t_{U^{(j)}}$ we get, restricting $\tilde{y}$ to $\Gamma_{s}^{(j)}$, that

$$
t_{U^{(j)}}(\tilde{y})=a_{j}+\tilde{y}^{-p}\left[\hat{g}(\tilde{y})+\tilde{y}^{p} b_{j} \log \tilde{y}\right],
$$

for some $\hat{g} \in \mathcal{O}_{\Gamma_{s}}^{*}, a_{j}, b_{j} \in \mathbf{C}, j=1,2$.

It follows that the restriction of $t_{U^{(j)}}, j=1,2$, to any sector in $\Gamma_{s}^{(j)}$ with vertex at $s \in S$ and small angle $\left(<\frac{2 \pi}{p}\right)$ is injective.

The class of affine coordinates introduced in (c) control the way the other coordinates that come from (a) and (b) approach $S$.

Let $N \subset \mathbf{C P}(2)$ be the set where the construction in (a) and (b) applies, and $\widetilde{N}$ its universal covering space, $\alpha: \widetilde{N} \rightarrow N$ the associated projection. The foliation $\alpha^{*} \mathcal{F}$ has a holomorphic first integral $\mathcal{F}$ ("multiform first integral for $\mathcal{F}$ ") defined as follows:

(i) we cover $\widetilde{N}$ by the open sets $\left\{\alpha^{-1}(U)\right\}_{U}, U$ as in (a), (b) and (c), and consider the transverse affine structure given by

$$
\left\{\left(\alpha^{-1}(U), t_{U} \circ \alpha\right)\right\}_{U}=\left\{\left(\widetilde{U}_{j}, t_{\widetilde{U}_{j}}\right)\right\}_{j}
$$

(ii) we fix a component of $\alpha^{-1}\left(U_{0}\right)$ with the corresponding point of $\alpha^{-1}\left(U_{0}\right)$ with the corresponding point of $\alpha^{-1}\left(p_{0}\right)\left(p_{0} \in U_{0}\right)$, also denoted by $U_{0}$ and $p_{0}$ in order to simplify the notation. Let $\tilde{p} \in \widetilde{N}$ and $\eta_{p_{0} \tilde{p}}$ be a curve which joins $p_{0}$ to $\tilde{p}$. Then we cover $\eta_{p_{0} \tilde{p}}$ by a sequence $U_{0}=\widetilde{U}_{0}, \widetilde{U}_{1}, \ldots, \widetilde{U}_{n}$ such that $\widetilde{U}_{j} \cap \widetilde{U}_{n} \neq \phi, j=0, \ldots, n-1$. We define $\left.F\right|_{\widetilde{U}_{1}}=c_{\widetilde{U}_{0} \widetilde{U}_{1}} t_{\widetilde{U}_{1}}+d_{\widetilde{U}_{0} \widetilde{U}_{1}}$, $\left.F\right|_{\widetilde{U}_{2}}=c_{\widetilde{U}_{0}, \widetilde{U}_{1}}\left(c_{\widetilde{U}_{1} \widetilde{U}_{2}} t_{\widetilde{U}_{2}}+d_{\widetilde{U}_{1} \widetilde{U}_{2}}\right)+d_{\widetilde{U}_{0} \widetilde{U}_{1}}, \ldots$ etc.

It is easily seen that the definition depends on $\eta_{p_{0} \tilde{p}}$ and on the sequence chosen to cover it (once we have fixed $\widetilde{U}_{0}$ ).

In the sequel we will fix $\widetilde{U}_{0}=U_{0}$ as one of the components of $\alpha^{-1}\left(U_{0}\right)$ such that $\bar{U}_{0} \cap S \neq \phi$ (see (c) above). 
We present now an application of the existence of the transverse affine structure such as constructed before. Let $s_{0} \in S$ be a regular point of $\mathcal{F}, s_{0} \in \partial U_{0}$ and $\gamma_{1}, \gamma_{2}$ be two closed curves in $\pi_{1}\left(S_{1}=S \backslash\left\{p_{0}, \ldots, p_{m}\right\}, s_{0}\right)$, where $p_{0}, \ldots, p_{m}$ are the singularities of $\mathcal{F}$ along $S$. Let also $h_{1}, h_{2}$ be the germs of holomorphic diffeomorphisms associated to $\gamma_{1}, \gamma_{2}$ as elements of the holonomy group $\mathcal{H}$ of $S_{1}$ defined in a section $\Gamma_{s_{0}}$ to $S$.

Theorem 2. If $\gamma_{1} * \gamma_{2}=\gamma_{2} * \gamma_{1}$ in $\pi_{1}\left(N, s_{0}\right)$, then $h_{2} \circ h_{1}=h_{1} \circ h_{2}$ in $\mathcal{H}$.

Proof. Let $\gamma=\gamma_{1} * \gamma_{2} * \gamma_{1}^{-1} * \gamma_{2}^{-1}$ and $h \neq h_{2}^{-1} \circ h_{1}^{-1} \circ h_{2} \circ h_{1}$. We assume that $h \neq$ id. Then there exists $\bar{y} \in \Gamma_{s_{0}}$ such that the sequence $\left\{h^{l}(\bar{y})\right\}_{l \in \mathbf{N}}$ goes to $0 \in \Gamma_{s_{0}}$ as $l \rightarrow \infty$ and the elements of this sequence are contained in a sector of small angle centered at $0 \in \mathbf{C}$ (since $h$ is tangent to Id). Now we define a first integral $F$ starting from an open set $U_{0}$ as in (c) and $p_{0}=\bar{y}$, according to the construction given above. For $n \in \mathbf{N}$, we lift $\gamma^{n}=\gamma * \cdots * \gamma(n$ times) to the leaf of $\mathcal{F}$ passing through $\bar{y}$ and get $\bar{\gamma}^{n} \subset N$ close to $S$ which joins $\bar{y}$ to $h^{n}(\bar{y})$. When we go up to the covering space $\widetilde{N}, \alpha^{-1}\left(\bar{\gamma}^{n}\right)$ joins $\bar{y}$ to some point $\tilde{y}_{n} \in \alpha^{-1}\left(h^{n}(\bar{y})\right)$; since $\gamma=1$ as an element of $\pi_{1}\left(N, s_{0}\right)$, this point belongs to $U_{0} \forall n \in \mathbf{N}$, so that we may take $\eta_{\bar{y} \tilde{y}_{n}}$ inside $U_{0}$; therefore we need only to use $U_{0}$ to cover $\eta_{\bar{y} \tilde{y}_{n}}$, which implies $F=t_{U_{0}}$ in $U_{0}$.

Since $F(\bar{y})=F\left(h^{l}(\bar{y})\right) \forall l \in \mathbf{N}$, it follows that $t_{U_{0}}(\bar{y})=t_{U_{0}}\left(h^{l}(\bar{y})\right) \forall l \in \mathbf{N}$, contradicting the injectivity of $t_{U_{0}}$ in sectors of angle $<\frac{2 \pi}{p}$.

\section{Partial Classification}

Let us consider again the examples of $\S 1$, and make a few remarks about the singularities of the foliations $\mathcal{F}_{1}$ and $\mathcal{F}_{p}$ (these foliations are presented in affine coordinates $\left.(x, y) \in \mathbf{C}^{2}\right)$.

In the case of example (i), we may assume that all singularities of $\left.\mathcal{F}_{i}\right|_{L}$ are simple; it is enough to choose $\lambda_{k} \notin \mathbf{R}_{+} \cup\{0\}, k=0, \ldots, \nu$. The same occurs in example (ii) with relation to the singularities $\left(a_{k}, 0\right), k=0, \ldots, \nu$. Now, if $\sum_{k=0}^{\nu} \lambda_{k}=1$, the point at infinity of $L$ is a dicritical singularity of $\mathcal{F}_{p}, p \geq 2$, although it is a regular point of $\mathcal{F}_{1}$. Examples with only simple singularities can be found if we demand $1-\sum_{k=0}^{\nu} \lambda_{k} \notin \mathbf{R}_{+} \cup\{0\}$ and $\operatorname{deg}(P)+p<\nu$, as an easy computation shows. The line at infinity will be also a separatrix of $\mathcal{F}_{p}$, and $\operatorname{deg}\left(\mathcal{F}_{p}\right)=\nu+1\left(\left.\mathcal{F}_{p}\right|_{L}\right.$ has $\nu+1$ simple singularities).

In all cases it is important to observe that the intersection point of the vertical separatrices is a dicritical singularity of $\mathcal{F}_{p}$ (again an easy computation). Also, this point is not a nodal point for the union of straight lines which are separatrices. Each of the theorems to be stated below emphasizes one of these aspects.

Remark. A dicritical singularity, after a blowing-up, gives rise to a foliation for which the divisor is not invariant.

Let $\mathcal{B}$ be the set of foliations in $\mathbf{C} P(2)$, with some fixed degree $\nu \in \mathbf{N}, \nu \geq 2$, such that:

1) any $\mathcal{F} \in \mathcal{B}$ admits some smooth invariant algebraic curve $S$ which contains only simple singularities of $\mathcal{F}$, one at least being hyperbolic, and whose holonomy group is a non abelian solvable group;

2) if $q, \tilde{q} \in S$ are different singularities of $\mathcal{F}$, then the indices $\operatorname{ind}_{q}(\mathcal{F}, S)$ and $\operatorname{ind}_{\tilde{q}}(\mathcal{F}, S)$ are different numbers. 
From Theorem 1, we know that in convenient affine coordinates $(x, y) \in \mathbf{C}^{2}$ there exists a closed meromorphic 1-form $\eta$ such that $d \omega=\eta \wedge \omega$, where $\omega=0$ represents the foliation $\mathcal{F} \in \mathcal{B}$. Furthermore, the separatrices of $\mathcal{F}$ which pass through the singularities of $\left.\mathcal{F}\right|_{S}$ are algebraic curves because they are lines of poles of $\eta$; if $C$ is one of these separatrices, then

$$
\operatorname{Res}_{C}(\eta)=1-p \operatorname{ind}_{q}(\mathcal{F}, S)
$$

where $q \in S$ is any of the singularities in $C \cap S$ and $p \in \mathbf{N}$ is the ramification index associated to the solvable group. Now 2 ) ensures that $\# C \cap S=1$, or equivalently, $S$ and the separatrices of its singularities are all straight lines. Let $\mathcal{L}(\mathcal{F})$ denote the union of these straight lines, transversal to $S$. If $\mathcal{L}(\mathcal{F})$ contains only singular points of nodal type, the fundamental group of $\mathbf{C} P(2) \backslash \mathcal{L}(\mathcal{F})$ is an abelian group (this is a particular case of Deligne's Theorem, see [6]). From Theorem 2 we conclude that the holonomy group of $S$ is abelian, what is not the case (see Remark at $\S 5$ ). Therefore $\mathcal{L}(\mathcal{F})$ contains at least a singular point which is not of nodal type.

Remark. The existence of singular points of $\mathcal{L}(\mathcal{F})$ which are not of nodal type follows also from the results of [3].

Theorem 3. Let $\mathcal{F} \in \mathcal{B}$ and assume that any straight line in $\mathcal{L}(\mathcal{F})$ contains at most a singular point of $\mathcal{L}(\mathcal{F})$ which is not of nodal type. Then $\mathcal{F}$ is a Bernoulli foliation.

Let us define $\widetilde{\mathcal{B}} \subset \mathcal{B}$ adding an extra condition:

3) there are no relations of the type $p \sum_{k} \varepsilon_{k} \operatorname{ind}_{q_{k}}(\mathcal{F}, S)=n$, where $n \in\{-\nu$, $-(\nu-1), \ldots, 0, \ldots, \nu-1, \nu\}, \varepsilon_{k}=0$ or $\varepsilon_{k}=1,3 \leq \sum_{k} \varepsilon_{k}<\nu+1$.

Since $S$ is a straight line, we may choose affine coordinates such that $S$ becomes the line at infinity and $\omega=\omega_{0}+\cdots+\omega_{\nu-1}+\omega_{\nu}$, where $\omega_{j}, 0 \leq j \leq \nu$, are polynomial homogeneous 1 -forms and

$$
\omega_{\nu}=\left(y-a_{0} x\right) \cdots\left(y-a_{\nu} x\right) \sum_{k=0}^{\nu} \lambda_{k} \frac{d\left(y-a_{k} x\right)}{y-a_{k} x} .
$$

The singularities $q_{0}, \ldots, q_{\nu}$ of $\left.\mathcal{F}\right|_{S}$ are the intersections of $\overline{\left\{u-a_{k} x=0\right\}}$ with $S$, $0 \leq k \leq \nu$, and $\operatorname{ind}_{q_{k}}(\mathcal{F}, S)=\lambda_{k}, 0 \leq k \leq \nu$.

Theorem 4. Let $\mathcal{F} \in \widetilde{\mathcal{B}}$ and assume that the singularities of $\mathcal{F}$ along $\mathcal{L}(\mathcal{F})$ are simple or dicritical singularities. Then $\mathcal{F}$ is a Bernoulli foliation.

The reader should notice that Theorem 3 deals with the number of singular points of $\mathcal{L}(\mathcal{F})$ which are not of nodal type, whereas the hypothesis of Theorem 4 associates more closely these points to the foliation. Clearly singular points of $\mathcal{L}(\mathcal{F})$ which are not of nodal type cannot be simple singularities of $\mathcal{F}$.

The main point in proving both theorems is to show that all straight lines in $\mathcal{L}(\mathcal{F})$ meet together at the same point. Once this is done, the following argument finishes the proofs. Let $S=\overline{\{y=0\}}, q_{0}, \ldots, q_{\nu}$ be the singularities of $\left.\mathcal{F}\right|_{S}$ and $C_{0}, \ldots, C_{\nu}$ their separatrices, where $C_{k}=\overline{\left\{x-q_{k}=0\right\}}, k=0, \ldots, \nu$ (since $C_{0}, \ldots, C_{\nu}$ meet at the same point, it is easy to pick up coordinates where they are all vertical lines). Therefore we may write $\eta=(p+1) \frac{d y}{y}+\sum_{k=0}^{\nu} \mu_{k} \frac{d x}{x-q_{k}}$, where $\mu_{k}=\operatorname{Res}_{C_{k}}(\eta)$. Let 
$\omega=P d y-Q d x=0$ represent $\mathcal{F}$. We have:

$$
\begin{gathered}
d \omega=\left(P_{x}+Q_{y}\right) d x \wedge d y \\
\eta \wedge \omega=\left[(p+1) \frac{Q}{y}+P \sum_{k=0}^{\nu} \frac{\mu_{k}}{x-q_{k}}\right] d x \wedge d y
\end{gathered}
$$

so that $P_{x}+Q_{y}=(p+1) \frac{Q}{y}+P \sum_{k=0}^{\nu} \frac{\mu_{k}}{x-q_{k}}$.

We see at once, since $P$ is a polynomial, that $P(x, y)=c\left(x-q_{0}\right) \cdots\left(x-q_{\nu}\right)$, for some $c \in \mathbf{C}^{*}$ (remember that $\left.\operatorname{deg} P \leq \nu+1\right)$.

It follows that $Q_{y}-(p+1) \frac{Q}{y}=R(x)$, where $R$ is a polynomial and so $Q(x, y)=$ $\frac{y}{p} \int R(x)+y^{p+1} B(x)$, for some polynomial $B$.

\section{Proof of Theorem 3}

Let $C_{0}, \ldots, C_{\nu}$ be the separatrices of $\mathcal{F} \in \mathcal{B}$ transversal to $S$, and suppose $C_{0}, \ldots, C_{\bar{\mu}}$ contain singular points of $\mathcal{L}(\mathcal{F})$ which are not of nodal type. We distribute these straight lines in classes $F_{0}, \ldots, F_{\bar{l}}$ such that in each $F_{j}, 0 \leq j \leq \bar{l}$, all straight lines meet at the same point; we have then a partition of the set $\left\{C_{0}, \ldots, C_{\bar{\mu}}\right\}$, by hypothesis. Finally we consider the classes $F_{\bar{l}+1}, \ldots, F_{l}$ containing each a single line taken among $C_{\bar{\mu}+1}, \ldots, C_{0}$.

Let $N=\mathbf{C} P(2) \backslash C_{0} \cup \cdots \cup C_{\nu}$ and $s_{0} \in S \cap N$; we take small circles $\gamma_{k}^{\prime} \subset S$ around each point $q_{k}$ and join $s_{0} \in S$ to points $q_{0}^{\prime}, \ldots, q_{\nu}^{\prime}$ contained in these circles, in such a way that the $\operatorname{arcs} \widehat{s}_{0} q_{k}^{\prime} * \gamma_{k}^{\prime} * \widetilde{q_{k}^{\prime} s_{0}}, 0 \leq k \leq \nu$, are generators for $\pi_{1}\left(N, s_{0}\right)$ (there are possibly other relations besides the obvious one $\gamma_{0} * \cdots * \gamma_{\nu}=1$ ).

Claim. If $q_{k}, q_{k^{\prime}}$ belong to straight lines in different classes, then $\gamma_{k} * \gamma_{k^{\prime}}=\gamma_{k^{\prime}} * \gamma_{k}$ as elements of $\pi_{1}\left(N, s_{0}\right)$.

This is obvious when $q_{k}, q_{k^{\prime}}$ and $C_{k} \cap C_{k^{\prime}}$ belong to some small neighborhood of $C_{k} \cap C_{k^{\prime}}$ disjoint from the other straight lines in $\mathcal{L}(\mathcal{F})$ (the fundamental group of the complement of $C_{k} \cup C_{k^{\prime}}$ in this neighborhood is isomorphic to $\mathbf{Z} \times \mathbf{Z}$ ). In general, the configuration of lines in $\mathcal{L}(\mathcal{F})$ can be brought to this particular one through an isotopy of $\mathbf{C} P(2)$ starting with the identity (see also [6]).

As a consequence of this claim, we cannot have more than one class in our partition, that is, $l=1$, and therefore the separatrices $C_{0}, \ldots, C_{\nu}$ meet together at the same point. In fact, suppose for simplicity we have two classes $F_{0}, F_{1}$. We fix a straight line $C \in F_{0}$, to which it is associated a local diffeomorphism $h_{0} \in \mathcal{H o l}\left(S \backslash\left\{q_{0}, \ldots, q_{\nu}\right\}, s_{0}\right)$. The elements of $\mathcal{H o l}\left(S \backslash\left\{q_{0}, \ldots, q_{\nu}\right\}, s_{0}\right)$ associated to the lines in $F_{1}$ commute with $h_{0}$, by Theorem 2 . It follows, since they belong to a subgroup of $\mathbf{H}_{p}$, that they commute among themselves. Reverting the argument, the elements of $\mathcal{H o l}\left(S \backslash\left\{q_{0}, \ldots, q_{\nu}\right\}, s_{0}\right)$ which correspond to straight lines in $F_{1}$ commute, and also commute with any element that comes from the lines in $F_{0}$. Therefore $\mathcal{H o l}\left(S \backslash\left\{q_{0}, \ldots, q_{\nu}\right\}, s_{0}\right)$ is abelian, contradiction.

\section{Proof of Theorem 4}

We already know that there must exist some singular point $p \in \mathcal{L}(\mathcal{F})$ which is not of nodal type; as a singularity of $\mathcal{F}$, this point is necessarily a dicritical singularity. Let us take affine coordinates such that $p=(0,0)$ and $S$ is the line at infinity; also, if $\omega=0$ represents $\mathcal{F}$, then $\omega=\omega_{m}+\cdots+\omega_{\nu}$, where $\omega_{m}, \ldots, \omega_{\nu}$ are homogeneous polynomial 1 -forms of degree $m, \ldots, \nu$, with $m \geq 1$. Let us 
suppose that $C_{0}, \ldots, C_{l}$ contain $p \in \mathbf{C} P(2)$ and $C_{j}=f_{j}^{-1}(0)=\left\{y-\alpha_{j} x=0\right\}$, $0 \leq j \leq l$, and the other lines $C_{l+1}, \ldots, C_{\nu}$ are given by $f_{j}=y-\alpha_{j} x-\beta_{j}, \beta_{j} \neq 0$, $l+1 \leq j \leq \nu$. Then $\eta_{0}=\sum_{t=0}^{\nu} \mu_{k} \frac{d f_{k}}{f_{k}}$; we define $\eta_{0}=\sum_{k=0}^{l} \mu_{k} \frac{d f_{k}}{f_{k}}$. From $d \omega=\eta \wedge \omega$ we get $d \omega_{m}=\eta_{0} \wedge \omega_{m}$. Putting $\omega_{m}=A(x, y) d x+B(x, y) d y$, where $A$ and $B$ are homogeneous polynomials of degree $m \in \mathbf{N}$ :

$$
\begin{aligned}
\omega_{m} & =x^{m}[A(1, t) d x+B(1, t) d(t x)] \\
& =x^{m}[(A(1, t)+t B(1, t)) d x+B(1, t) x d t] \\
& =x^{m+1} B(1, t) d t,
\end{aligned}
$$

because $(0,0)$ is a dicritical singularity. Therefore

$$
\begin{aligned}
(m+1) B(1, t) x^{m} d x \wedge d t & =\sum_{k=0}^{l} \mu_{k} \frac{d\left(\left(t-\alpha_{k}\right) x\right)}{\left(t-\alpha_{k}\right) x} \wedge\left(x^{m+1} B(1, t) d t\right) \\
& =\left(\sum_{k=0}^{l} \mu_{k}\right) x^{m} B(1, t) d x \wedge d t
\end{aligned}
$$

so that $\sum_{k=0}^{l} \mu_{k}=m+1$.

But $\mu_{k}=1-p \lambda_{k}$, so $(l+1)-p \sum_{k=0}^{l} \lambda_{k}=m+1$ and $p \sum_{k=0}^{l} \lambda_{k}=l-m$. We conclude from condition (3) that $l=\nu$.

\section{Remarks.}

(1) We pointed out earlier that when the algebraic separatrix $S \subset \mathbf{C P}(2)$ has abelian holonomy group (also the singularities of $\mathcal{F}$ along $S$ are simple and one of them is hyperbolic), a meromorphic integrating factor $f$ can be constructed for the polynomial 1-form $\omega$ that defines $\mathcal{F}$. It follows immediately that $d \omega=$ $\eta \wedge \omega$ where $\eta=\frac{d f}{f}$ and $\omega=P_{0} \cdots P_{m} \sum_{j=0}^{m} \mu_{j} \frac{d P_{j}}{P_{j}}$, where $P_{0}=0, \ldots, P_{m}=0$ are the equations for $S$ and its separatrices (see [1]). The multivalued function $P_{0}^{\mu_{0}} \cdots P_{m}^{\mu_{m}}$ is a Darboux integral for $\mathcal{F}$.

The (dynamical) hypothesis "there exists a (transcendental) leaf which accumulates only on the algebraic set $P_{0} \cdots P_{m}=0$ " guarantees the commutativity of the holonomy group of $S$.

(2) We proved that the holonomy group of $S$ is an abelian group when there exists a meromorphic closed 1-form $\eta$ such that $d \omega=\eta \wedge \omega$ and $(\eta)_{\infty}$ is a normal crossings divisor.

(3) Let us write $\eta=\sum_{j=0}^{m} \lambda_{j} \frac{d P_{j}}{P_{j}}(d \omega=\eta \wedge \omega)$.

Proposition. If $\lambda_{0} \in\{2,3, \ldots\}$ the holonomy group of $S=\left\{P_{0}=0\right\}$ is solvable; if $\lambda_{0} \notin\{2,3, \ldots\}$ it is abelian.

Proof. Assume first that $\lambda_{0} \in \mathbf{N}^{*}$. Let us consider the affine transversal structure associated to $\mathcal{F}$ (the notation is the same as before); let also $s_{0} \in S$ be a regular point of $\mathcal{F}$. There exists a neighborhood of $s_{0}, U=D \times \Gamma\left(D \subset S\right.$ is a disc, $\Gamma \ni s_{0}$ is transversal to $S$ ), where $\eta=\frac{d\left(y^{\lambda_{0}}\right)}{y^{\lambda_{0}}}$ for some appropriate coordinate $y \in \Gamma$, $y\left(s_{0}\right)=0$. When $y \in \Gamma$ is restricted to a sector $V$ of vertex $s_{0}$, one has $\frac{1}{y^{\lambda_{0}}} \omega=d t_{V}$ for some $t_{V} \in \mathcal{O}_{D \times V}$. Along $\Gamma, \omega=a(y) d y, a \in \mathcal{O}_{\Gamma}, a(0) \neq 0$. 
Case 1. $\lambda_{0} \in\{2,3, \ldots\}$. Let $h \in \mathcal{H}_{0}$, the subgroup of elements of $\mathcal{H}$ which are tangent to the identity. Since $h$ is associated to the lifting of a closed curve in $S$ with $s_{0} \in S$ as base point, we have

$$
t_{V}(y)=A t_{V}(h(y))+B, \quad A \in \mathbf{C}^{*}, B \in \mathbf{C},
$$

whenever $h(V) \subset V$. Differentiating $(*)$ :

or

$$
\frac{d t_{V}(y)}{d y}=A \frac{d t_{V}}{d y}(h(y)) \cdot h^{\prime}(y)
$$

$$
\frac{a(y)}{y^{\lambda_{0}}}=A \frac{a(h(y))}{h(y)^{\lambda_{0}}} \cdot h^{\prime}(y)
$$

so that

$$
A=\frac{a(y)}{a(h(y))}\left(\frac{h(y)}{y}\right)^{\lambda_{0}} \cdot \frac{1}{h^{\prime}(y)} .
$$

Letting $y \rightarrow 0$ yields $A=1$.

The conclusion is: in the sector $V, h$ is given by the translation $t_{V} \mapsto T_{V}-B$.

We want to prove that $\mathcal{H}_{0}$ is abelian. Suppose that $h, \tilde{h} \in \mathcal{H}_{0}$ are such that $h_{1}=[h, \tilde{h}] \neq \mathrm{id}$. The sequence $h_{2}=\left[h, h_{1}\right], \ldots, h_{n}=\left[h, h_{n-1}\right], \ldots$ has increasing orders of tangency with the identity, so that we may eventually find $h_{n}$ with an invariant sector $V^{\prime} \subset V\left(h_{n}\left(V^{\prime}\right) \subset V^{\prime}\right)$; in $V^{\prime}$ we have $h_{n}\left(t_{V}\right)=t_{V}-B^{\prime}$ for $t_{V} \in V^{\prime}$, so that $\left[h, h_{n}\right]=\mathrm{id}$, contradiction.

Case 2. $\lambda_{0}=1$. We have that $t_{V}(y)=c \log y+b(y)$, for some $b \in \mathcal{O}_{\Gamma}, c \neq 0$, so that

$$
c \log y+b(y)=A[c \log h(y)+b(y)]+B
$$

follows in some sector $V$ invariant for $h \in \mathcal{H}_{0}(h(V) \subset V)$. Therefore $c \log y-$ $A c \log h(y)=b(y)-A b(h(y))-B$ is holomorphic at $0 \in \mathbf{C}$, yielding $A=1$ and $B=0$. Since $t_{V}$ is injective when the angle of $V$ at $s_{0} \in \Gamma$ is small, we conclude that $h(y) \equiv y$.

Case 3. $\lambda_{0}=-m, m \in \mathbf{N}^{*}$. Since $t_{V} \in \mathcal{O}_{\Gamma}$ in this case, $(*)$ applied to $h \in \mathcal{H}_{0}$ implies that $A=1$ and $B=0$. Again the injectivity of $t_{V}$ when $V$ has small angle at $s_{0} \in \Gamma$ leads to $h(y) \equiv y$.

Finally we assume that $\lambda_{0} \notin \mathbf{R}$. From (*) we have $\frac{a(y)}{y^{\lambda_{0}}}=A \frac{a(h(y))}{h(y)^{\lambda} 0} h^{\prime}(y)$, which implies that $\left(\frac{h(y)}{y}\right)^{\lambda_{0}}=A \frac{a(h(y))}{a(y)} h^{\prime}(y)$. The function $\left(\frac{h(y)}{y}\right)^{\lambda_{0}}$ would have a holomorphic extension to $y=0$, unless $h(y) \equiv y$.

(4) Let $\mathcal{F} \in \mathcal{B}$ (see $\S 3$ ) and $\mathcal{F}^{\prime}$ be a foliation close to $\mathcal{F}$ such that the straight line $S$ is invariant for $\mathcal{F}$ and $\mathcal{F}^{\prime}$, contains $\nu+1$ singularities and has solvable holonomy. Then it is easily seen that all the conditions of Theorem 3 (or Theorem 4) are still valid for $\mathcal{F}^{\prime}$.

\section{APPENDIX}

We prove here Theorem 1 for the reader's convenience. We fix affine coordinates $(x, y) \in \mathbf{C}^{2}$ such that $L_{\infty}=\mathbf{C} P(2) \backslash \mathbf{C}^{2}$ is not invariant, $S \cap L_{\infty}, \operatorname{Sing}(\mathcal{F}) \cap S \subset \mathbf{C}^{2}$. As we said before, the holonomy group $\mathcal{H}$ of $S \backslash \operatorname{Sing}(\mathcal{F})$ is conjugated, according to [5], to a subgroup $H \subseteq \mathbf{H}_{p}$, for some $p \in \mathbf{N}$. As a matter of fact, $\mathcal{H}$ depends on 
the transversal section to $S$ as we choose to define it, but we take for granted that $H$ is fixed once and for all.

The idea of the proof is to construct the 1-form $\eta$ in a neighborhood of $S$; then by Levi's theorem (see [1]) it extends to $\mathbf{C} P(2)$ as a meromorphic 1-form. There are several cases to be considered.

(1) Let $s \in S \backslash \operatorname{Sing}(\mathcal{F}), s \notin L_{\infty}$. We take $\Gamma, \Gamma^{\prime}$ transverse sections to $S$ contained in a neighborhood of $s \in S$, and $z, z^{\prime} \in \mathbf{C}$ complex coordinates for $\Gamma, \Gamma^{\prime}$ which conjugate $\mathcal{H}_{\Gamma}$ and $\mathcal{H}_{\Gamma^{\prime}}$ to $H$. It is easy to see that $z^{\prime}=\frac{a z}{\sqrt[p]{1+b z^{p}}}$ for some $a \neq 0, b \in \mathbf{C}$, so we have $\frac{d z^{\prime}}{\left(z^{\prime}\right)^{p+1}}=\frac{1}{a^{p}} \frac{d z}{z^{p+1}}$.

Since we can represent locally $\mathcal{F}$ as $z^{-(p+1)} d z=0$ or $\left(z^{\prime}\right)^{-(p+1)} d z^{\prime}=0$, there exist meromorphic functions $h, h^{\prime}$ defined in a neighborhood of $s \in \mathbf{C}$ satisfying

$$
\omega=h \frac{d z}{z^{p+1}}=h^{\prime} \frac{d z^{\prime}}{\left(z^{\prime}\right)^{p+1}}
$$

It follows that $h^{\prime}=a^{p} h$ and $\frac{d h^{\prime}}{h^{\prime}}=\frac{d h}{h}$, and we may define $\eta:=\frac{d h}{h}=\frac{d h^{\prime}}{h^{\prime}}$. We have then

$$
d \omega=d h \wedge \frac{d z}{z^{p+1}}=\frac{d h}{h} \wedge\left(h \frac{d z}{z^{p+1}}\right)=\eta \wedge \omega
$$

In this way we define $\eta$ in a neighborhood of $(S \backslash \operatorname{Sing}(\mathcal{F})) \cap \mathbf{C}^{2}$. The problem now is to extend $\eta$ to $\operatorname{Sing}\left(\left.\mathcal{F}\right|_{S}\right)$ and to $S \cap L_{\infty}$.

(2) Let $p_{j} \in S \cap \operatorname{Sing}(\mathcal{F})$; we assume that locally $p_{j}=(0,0)$ and $\omega=x d y-$ $\lambda y d x+$ h.o.t., $S=\{y=0\}$. In fact, when $\lambda \notin \mathbf{R}$, or $\lambda \in \mathbf{R}_{-}$but $\lambda \notin \mathbf{Q}_{-}$we may assume that $\omega=x d y-\lambda y d x$ (since the local holonomy $h$ of $S$ around $p_{j}$ belongs to $\mathbf{H}_{p}$ it can be made linear in some appropriate coordinate; by $[9], \omega$ can be also made linear).

Let us take a transverse section $\Gamma$ to $S$ close to $p_{j}$; for simplicity we assume that $\Gamma=\left\{\left(1, y_{0}\right) ; y_{0} \in \mathbf{C}\right\}$.

Claim. If $z \in \Gamma$ conjugates $\mathcal{H}_{\Gamma}$ to $\mathcal{H}$, then

$$
z=\frac{\alpha y_{0}}{\sqrt[p]{1+\beta y_{0}^{p}}}, \quad \text { for some } \alpha \neq 0, \beta \in \mathbf{C}
$$

In order to prove the claim, we follow the diagram; here $z=\xi\left(y_{0}\right)$ ( $\xi$ is to be determined!),

$$
\begin{gathered}
h(z)=\frac{e^{2 \pi i \lambda} z}{\sqrt[p]{1+\zeta z^{p}}}, \quad \tilde{z}=\pi(z)=z^{p}, \quad \tilde{h}(\tilde{z})=\frac{\left(e^{2 \pi i \lambda}\right)^{p} \tilde{z}}{1+\beta \tilde{z}} \\
\tilde{l}(u)=\left(e^{2 \pi i \lambda}\right)^{p} u, \quad l\left(y_{0}\right)=e^{2 \pi i \lambda} . y_{0}, \quad u=M(\tilde{z})
\end{gathered}
$$


where $M$ is the Moebius transformation, $\left(M^{\prime}(0)=1\right)$ which conjugates $\tilde{h}$ to $\tilde{l}$.

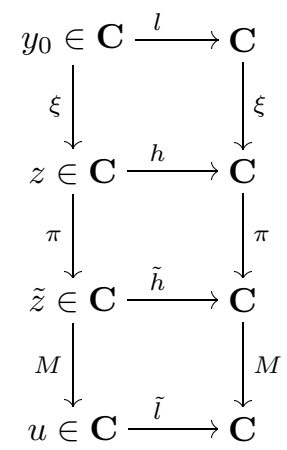

We have then $\tilde{l} \circ M \circ \pi \circ \xi=M \circ \pi \circ \xi \circ l$, or

$$
\left(e^{2 \pi i \lambda}\right)^{p} \cdot M\left(\xi\left(y_{0}\right)^{p}\right)=M\left(\xi\left(e^{2 \pi i \lambda} y_{0}\right)^{p}\right) .
$$

If $\theta:=e^{2 \pi i \lambda}, f\left(y_{0}\right):=\xi\left(y_{0}\right)^{p}$, we get

$$
\theta^{p} \cdot M\left(f\left(y_{0}\right)\right)=M\left(f\left(\theta y_{0}\right)\right) \quad \text { or } \quad \theta^{p} \hat{f}\left(y_{0}\right)=\hat{f}\left(\theta y_{0}\right),
$$

where $\hat{f}:=M \circ f$.

A computation using power series (and the fact that $\theta$ is not a root of unity) yields $\hat{f}(y)=\alpha^{p} y_{0}^{p}$ for some $\alpha \neq 0$, and so $\xi\left(y_{0}\right)^{p}=\frac{\alpha^{p} y_{0}^{p}}{1+\beta y_{0}^{p}}$. The claim is proved.

As a consequence we get

$$
\frac{d z}{z^{p+1}}=\frac{1}{\alpha^{p}} \frac{d y_{0}}{y_{0}^{p+1}}
$$

But $y_{0}=y x^{-\lambda}$ is the equation for the leaf of $\omega=0$ which passes through $\left(1, y_{0}\right)$, so that $d y_{0}=x^{-\lambda-1} \omega$, and then

$$
\frac{d z}{z^{p+1}}=\frac{1}{\alpha^{p} y^{p+1} x^{-\lambda p+1}} \omega .
$$

It follows that

$$
\eta=\frac{d\left(\alpha^{p} y^{p+1} x^{-\lambda p+1}\right)}{\alpha^{p} y^{p+1} x^{-\lambda p+1}}=(p+1) \frac{d y}{y}+(1-\lambda p) \frac{d x}{x}
$$

in a neighborhood of $\Gamma$; one sees that $\eta$ extends to $p_{j}=(0,0)$ as $(p+1) \frac{d y}{y}+(1-\lambda p) \frac{d x}{x}$.

(3) Now let $\omega=x d y-\lambda y d x+$ h.o.t., $p_{j}=(0,0) \in \operatorname{Sing}(\mathcal{F}), S=\{y=0\}$ and $\lambda \in \mathbf{Q}_{-}(\lambda \neq 0)$. We study first the case where $\omega$ can be made linear in some coordinate system; in order to simplify the notation we write $\omega=$ $x d y+\frac{m}{n} y d x$. The same steps as in (2) lead to the equation

$$
\theta^{p} \hat{f}\left(y_{0}\right)=\hat{f}\left(\theta y_{0}\right),
$$

but $\theta$ is a root of unity $\left(\theta^{n}=1\right)$.

Again power series methods give

$$
\hat{f}\left(y_{0}\right)=\sum_{j=0}^{\infty} a_{j} y_{0}^{p+j n}=\alpha^{p} y_{0}^{p} \psi\left(y_{0}\right),
$$

where $\alpha \neq 0$ and $\psi\left(y_{0}\right)=\varphi\left(y_{0}^{n}\right)$ for some holomorphic function $\varphi$ defined at $0 \in \mathbf{C}$, $\varphi(0) \neq 0$. 
Therefore

$$
z^{p}=\frac{\alpha^{p} y_{0}^{p} \psi\left(y_{0}\right)}{1+\beta y_{0}^{p} \psi\left(y_{0}\right)}
$$

and

$$
\frac{d z}{z^{p+1}}=\frac{1}{\alpha^{p}}\left(\frac{1}{y_{0}^{p+1} \psi\left(y_{0}\right)}+\frac{\psi^{\prime}\left(y_{0}\right)}{y_{0}^{p} \psi\left(y_{0}\right)^{2}}\right) d y_{0} .
$$

Since $y_{0}=y x^{m / n}$ along the leaves of $\omega=0$, we find that

$$
\frac{d z}{z^{p+1}}=\frac{1}{\alpha^{p}}\left(\frac{1}{y_{0}^{p+1} \psi}+\frac{\psi^{\prime}}{y_{0}^{p} \psi^{2}}\right) x^{m / n-1} \omega
$$

so

$$
\eta=(p+1) \frac{d y}{y}+\left(1+\frac{m}{n} p\right) \frac{d x}{x}+\frac{d g}{g},
$$

where

$$
g(x, y)=\frac{\psi^{2}\left(y x^{m / n}\right)}{\psi\left(y x^{m / n}\right)+y x^{m / n} \psi^{\prime}\left(y x^{m / n}\right)} .
$$

From $\psi\left(y_{0}\right)=\varphi\left(y_{0}^{n}\right)$ it follows easily that $g$ is a holomorphic function without zeroes; the conclusion is that $\eta$ can be extended to $p_{j}$ as a meromorphic 1-form, and the polar set is $\{x=0\} \cup\{y=0\}$.

(4) Suppose now that $\omega=x d y-\lambda y d x+$ h.o.t., $\lambda \in \mathbf{Q}_{-}, S=\{y=0\}$ cannot be made linear at $p_{j}=(0,0)$. By hypothesis, the local holonomy map $h$ of $S$ around $p_{j}$, defined at a section $\Gamma \cap S$ is conjugated to a map $T \in \mathbf{H}_{p}$, with $T^{\prime}(0)=\alpha=e^{2 \pi i \lambda}$; there $T$ covers $\widetilde{T} \in \mathbf{H}, \widetilde{T}(0)=\alpha^{p}$. If $\alpha^{p} \neq 1, \widetilde{T}$ can be made linear in some coordinate, and consequently the same holds for $T$ and $h$; by a theorem of [9], $\omega=0$ can also be made linear in some coordinate system, contradiction. It follows that $\alpha^{p}=1$, or $\lambda=\frac{m}{p}$. Assuming $T: y_{0} \mapsto \frac{\alpha y_{0}}{\sqrt[p]{1-y_{0}^{p}}}$, and noticing that the equation $(*) p x d y+m y\left(1+\frac{i}{2 \pi} x^{m} y^{p}\right) d x=0$ has also $T$ as the local holonomy map around $(0,0)$, we conclude again by [9] that $\omega=0$ is conjugated to $(*)$. We write then $\omega=p x d y+m y\left(1+\frac{i}{2 \pi} x^{m} y^{p}\right) d x$ for simplicity. We have then the following situation: on one hand, $h=T$ in the section $\Gamma=\left\{\left(1, y_{0}\right), y_{0} \in \mathbf{C}\right\}$. On the other hand, the coordinate $z \in \mathbf{C}$ along $\Gamma$ that comes from (1) conjugates $h$ to an element of $H \subset \mathbf{H}_{p}$ which, modulo a conjugation in $\mathbf{H}_{p}$, can be assumed to be also $T$. Therefore, if $z=\xi\left(y_{0}\right)$, we have that $\xi$ belongs to the centralizer of $T$, so by [5] we have that $\xi \in \mathbf{H}_{p}: z=\xi\left(y_{0}\right)=\frac{\tilde{\alpha} y_{0}}{\sqrt[p]{1-\beta y_{0}^{p}}}$. We finish the argument as in (2).

(5) There remains to be analyzed only the extension of $\eta$ to $S \cap L_{\infty}=\left\{\bar{p}_{0}\right\}$. This is very simple, since we can give affine coordinates $u=\frac{1}{x}, v=\frac{y}{x}$ where $\bar{p}_{0}=(0,0), S=\{V=0\}$ and $L_{\infty}=\{u=0\} . \mathcal{F}$ is represented by $\tilde{\omega}=0$ when $u^{\nu+2} \omega=\tilde{\omega}$. From the previous construction we can write $d \tilde{\omega}=\tilde{\eta} \wedge \tilde{\omega}$, and therefore $\eta=\tilde{\eta}-(\nu+2) \frac{d u}{u}$. The 1 -form $\eta$ can be extended to $\bar{p}_{0}$ as $\tilde{\eta}-(\nu+2) \frac{d u}{u}$. 


\section{REFERENCES}

1. C. Camacho, A. Lins Neto and P. Sad, Foliations with Algebraic Limit Sets, Ann. of Math. 136 (1992). MR 93i:32035

2. C. Camacho and P. Sad, Invariant Varieties Through Singularities of Holomorphic Vector Fields, Ann. of Math. 115 (1982). MR 83m:58062

3. D. Cerveau and A. Lins Neto, Holomorphic Foliations in $\mathbf{C P}(2)$ having an Invariant Algebraic Curve, Ann. Inst. Fourier 41 (1991). MR 93b:32050

4. D. Cerveau et J-F. Mattei, Formes Intégrables Holomorphes Singulières, Astérisque 97, Soc. Math. France, 1982. MR 86f:58006

5. D. Cerveau et R. Moussu, Groupes d'Automorphismes de $(\mathbf{C}, 0)$ et Équations Différentielles $y d y+\cdots=0$, Bull. Soc. Math. France. 116 (1988). MR 90m:58192

6. P. Deligne, Le Groupe Foundamental du Complément d'une Courbe Plane n'ayant que des Points Doubles Ordinaires est Abélian, Sem. Bourbaki, Lecture Notes in Math. 842, Springer, 1981. MR 83f: 14026

7. I. Kaplansky, An Introduction to Differential Algebra, Hermann, Paris, 1957. MR 20:177

8. A. Lins Neto, Construction of Singular Holomorphic Vector Fields in Dimension Two, J. Differential Geometry 26 (1987). MR 88f:32047

9. J-F. Mattei et R. Moussu, Holonomie et Intégrales Premières, Ann. Sci. Ecole Norm. Sup. 13 (1980). MR 83b:58005

10. M. Prelle and M. Singer, Elementary First Integrals of Differential Equations, Trans. Amer. Math. Soc. 279 (1983), 215-229. MR 85d:12008

11. B. Scárdua, Transversely Affine Foliations on Complex Projective Spaces, IMPA, Thesis.

12. B. Seke, Sur les Structures Transversalement Affines des Feuilletages de Codimension Un, Ann. Inst. Fourier 30 (1980). MR 82b:57023

13. M. Singer, Liouvillian First Integrals of Differential Equations, Trans. Amer. Math. Soc. 333 (1992), 673-688. MR 92m:12014

14. J. Tits, Free Subgroups in Linear Groups, J. Algebra 20 (1972). MR 44:4105

Université de Rennes I - IRMar, Campus de Beaulieu - 35042, Rennes, France

Instituto de Matemática Pura e Aplicada (imPA), Estrada Dona Castorina 110, CEP 22460-320 Rio DE Janeiro, BraziL 